\title{
Activating Power of the Phosphopantetheine Arm in $\alpha$-Oxoamine Synthase Catalysis
}

\author{
Sarah E. Ackenhusen, Ye Wang, Stephanie W. Chun, Alison R. H. Narayan
}

\section{Abstract}

PLP-dependent enzymes are attractive biocatalysts due to their ability to perform complexity building transformations on amine-containing, readily available substrates from the chiral pool such as amino acids. Although many classes of PLPdependent enzymes are routinely employed in synthesis, select families remain underutilized due to limitations in practical scalability. For example, some PLP-dependent enzymes act on prohibitively expensive substrates such as coenzyme A-bound substrates. To address this challenge, we have investigated the mechanism of action of coenzyme $A$ in gating catalysis of one a-oxoamine synthase, SxtA AOS. Through investigation of the reactivity of SxtA AOS with panels of substrates and in the presence of various coenzyme $A$ mimics as well as monitoring PLP absorbances and the behavior of SxtA AOS variants, we have determined that activity is gated through the binding of the eastern phosphodiester group by the residue Arg142 that is located in a tunnel that leads to the active site. To circumvent this gating mechanism, a synthetic CoA mimic additive was designed that allows for enhanced reactivity with nonCoA substrates. These findings outline a strategy for employing AOS enzymes without the need for costprohibitive coenzyme A substrates.

\section{Introduction}

As highly functionalized molecules from the chiral pool, a-amino acids are valuable building blocks in

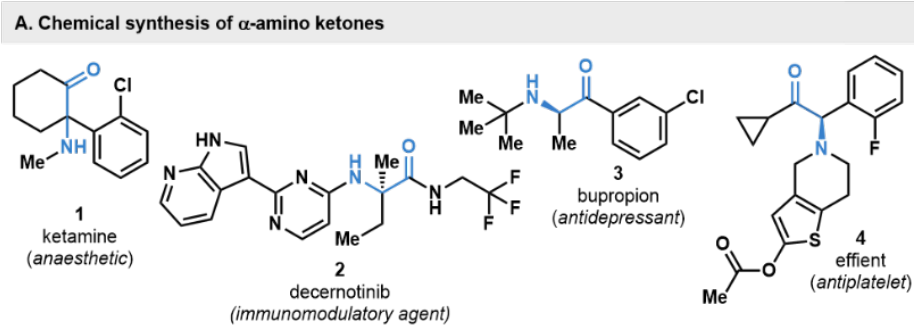

C. SxtA AOS native reactivity towards the neurotoxin saxitoxin synthesis. Building forward from a-amino acids allows for inclusion of several motifs commonly embedded in pharmaceutical agents, such as amine substituents and carboxylic acid groups, as well as diverse side chains that can provide multiple functional handles for diversification and an established stereocenter. ${ }^{1}$ Specifically, a-amino acids are a tantalizing starting point for the synthesis of $\alpha$-amino ketones, such as those shown in Figure 1A. Despite formally requiring breaking one bond and forming just one bond, synthetic approaches towards such motifs often require alternative starting materials as amino acids often present a chemoselectivity challenge, requiring protection and deprotection steps to furnish the desired target (Figure 1B). ${ }^{2-6}$

By contrast, the liability created by the need to protect the amino group for traditional methods is an asset in the biocatalytic elaboration of $\alpha$-amino acids. In particular, pyridoxal phosphate (PLP)-dependent enzymes leverage the amino group to condense onto the PLP cofactor and initiate catalysis (7, Figure 1B). ${ }^{7-}$ 10 PLP-dependent transaminases have become ubiquitous in biocatalytic syntheses, appearing in the synthesis of sitagliptin, among other compounds. ${ }^{8,11}$ Transaminases are popular due to their simplicity; Lamino acids and small molecule biologically relevant compounds are their substrates, which are often inexpensive starting materials whose simplified scaffolds are conducive to directed evolution. ${ }^{12-14}$ Racemases have also become valuable biocatalysts, for similar reasons. ${ }^{8,15}$ Perhaps an under-exploited

B. Transformation of $\alpha$-amino acids
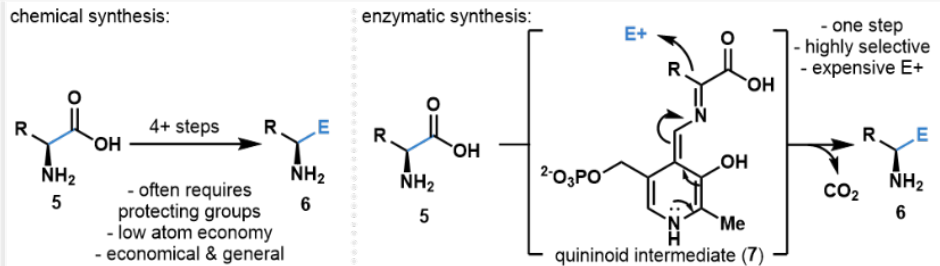

D. CoA gates productive catalysis

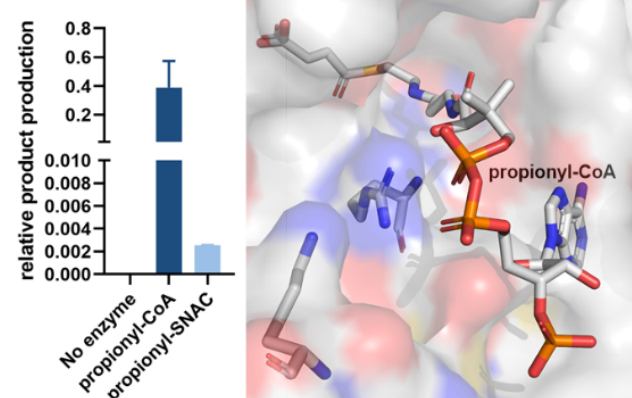

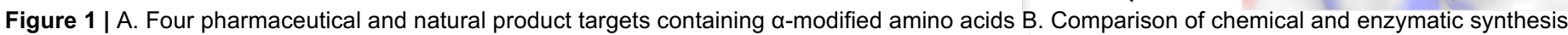

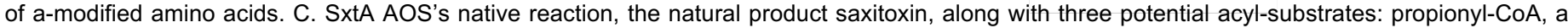

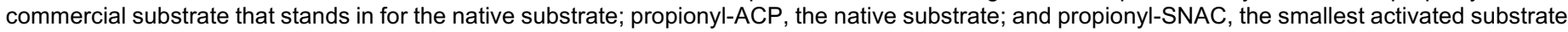

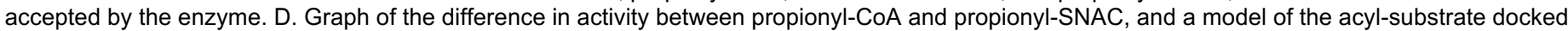
into SxtA AOS. AOS: a-oxoamine synthase, CoA: coenzyme-A, ACP: acyl-carrier protein, SNAC: N-acetylcysteamine 
enzymatic transformation is cabon-carbon bond formation, and one class of PLP-dependent enzyme that can mediate $\mathrm{C}-\mathrm{C}$ bond formation is the $\alpha$ oxoamine synthase (AOS). ${ }^{16}$ These catalysts rely on initial condensation of an amine substrate onto the PLP cofactor to generate an external aldimine intermediate. ${ }^{16}$ The $\mathrm{pK}_{\mathrm{a}}$ of the $\alpha$-proton is significantly lower in the aldimine intermediate, allowing for deprotonation by a proximal basic residue to form a nucleophilic quinonoid intermediate (see 7, Figure 1B) which can subsequently attack an electrophile. AOS reactions on a-amino acids are terminated by decarboxylation and stereoselective protonation to form products such as $\mathbf{6}$.

The potential of this $\mathrm{C}-\mathrm{C}$ bond-forming enzyme class is attenuated as AOSs require the electrophile to either be carrier protein-bound or a CoA-activated thioester (see Figure 1C). Carrier protein-bound acyl substrates require stoichiometric amounts of protein and pre-generation of the substrate loaded, holo protein. Alternatively, the use of free thioester substrates currently requires coenzyme A-activated acyl substrates for productive turnover, a reagent with high market value. An example of these limitations is highlighted in the transformation of L-arginine to ketone $\mathbf{8}$ with propionyl-CoA where a five-gram reaction would cost over $\$ 50,000$ for the propionyl-CoA alone.

The cost of coenzyme $A$ is therefore prohibitive for the use of AOSs as biocatalysts. To avoid the requirement for a stoichiometric CoA substrate or carrier protein-bound substrate, the role of the acyl substrate in gating catalysis must be more clearly understood. Previous studies have suggested two possible interactions between the enzyme and the thioester substrate: (1) with the carbonyl oxygen and (2) the sulfur atom. Ikushiro and coworkers previously explored the interaction of the carbonyl oxygen with an active site histidine of a serine palmitoyltransferase, another member of the AOS family. ${ }^{17,18}$ Rapid acceleration of deprotonation of Lserine was observed upon addition of palmitoyl-CoA or a thioether analog to the reaction. Based on kinetic studies and analysis of the crystal structure, Ikushiro asserts that a major factor in driving catalysis was the shift of this active site His (Supplementary Figure 4). This residue serves as a hydrogen bond partner for the carboxylate group of the amino acid substrate. Upon entering the active site, the acyl substrate is proposed to induce a shift in the nature of the histidine interactions, to interact with carbonyl oxygen of the acyl substrate. This change is proposed to position the amino acid for deprotonation and activate the carbonyl group of the acyl-CoA substrate for electrophilic attack. A second proposal surrounding the interaction of the acyl substrate and the enzyme focuses on a moiety outside the active site. ${ }^{19-23}$ Specifically, it is proposed that the aliphatic arm and the phosphodiester groups of CoA/ACP are involved in binding; ${ }^{19-23}$ however, no additional studies have defined the specifs of this binding event. Much of the research into this interaction occurs with overall binding studies of the acyl-CoA substrate, or observations of AOS crystal structures. ${ }^{19-23}$ Multiple research groups have identified the coenzyme $A$ substrate as essential for catalysis through kinetics studies with related enzymes, including 5aminolevulinate synthase, ${ }^{19} \mathrm{BioF},{ }^{24}$ and a serine palmitoyltransferase. ${ }^{25}$ Experiments led by Alexeev identified a possible interaction with the expensive electrophile by observing a concentration of positively charged residues proximal to the phosphate groups at the tail end of the acyl-coenzyme A substrate in a crystallographic study of BioF (PDB 1BSO). ${ }^{26}$

Understanding the role that the natural acyl substrate plays mechanistically is essential for engineering AOSs into effective catalysts. We anticipated that a detailed understanding of the acyl substrate-protein interaction could inform the engineering of a system that is CoA- or ACP-free and thus suitable for preparative-scale biocatalytic reactions.
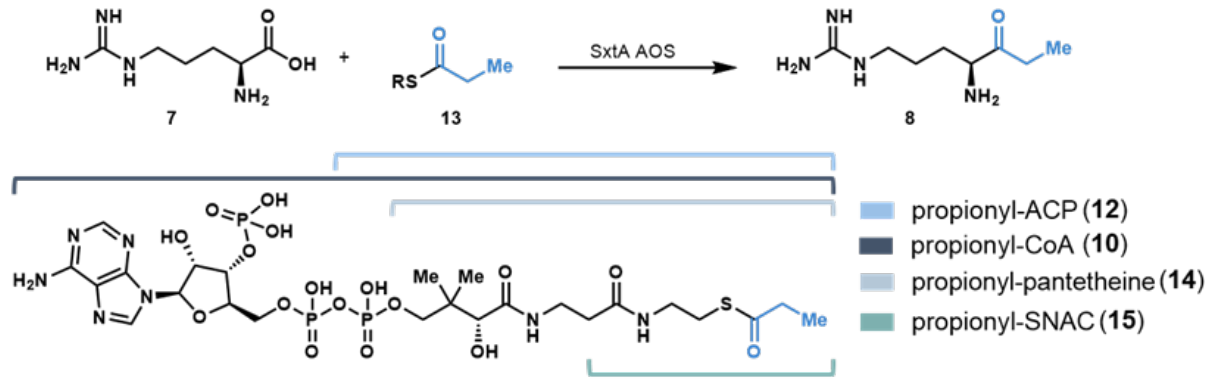

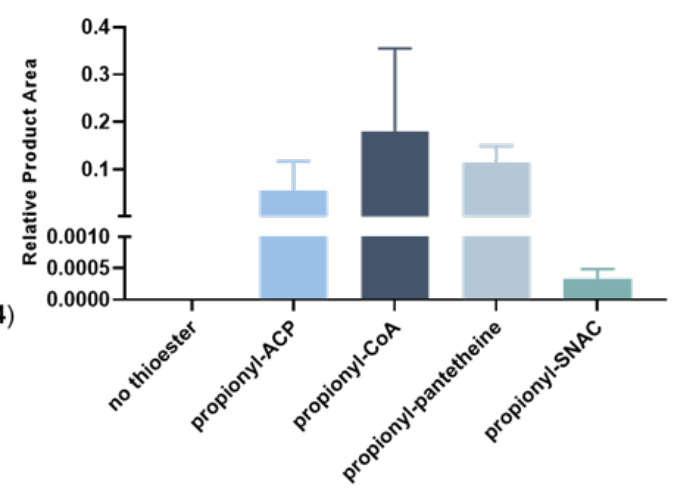

Figure 2 | Native acyl-substrate reactivity with wild-type enzyme. As the acyl substrate is truncated, activity decreases, as seen by HPLC-MS areas of the extracted ion chromatogram compared to tryptophan internal standard. 


\section{Results and discussion}

Toward understanding the role of the acyl substrate in gating AOS chemistry, we directed our focus to an AOS with demonstrated acyl substrate promiscuity. SxtA AOS is the last domain of a larger multidomain protein, SxtA, the first enzyme in the biosynthesis of saxitoxin (9, Figure 1C). ${ }^{27}$ This PLP-dependent enzyme transforms L-Arg into the corresponding ethyl ketone 8 with a propionyl group provided by the upstream ACP domain. In addition to acting on ACPbound acyl substrates, SxtA AOS readily accepts propionyl-CoA as a substrate (Figure 2). To assess the reactivity with various thioester substrates, initial experiments were performed with $20 \mu \mathrm{M}$ SxtA AOS, 2 $\mathrm{mM}$ of the native amino acid substrate L-Arg and 0.5 $\mathrm{mM}$ of four propionyl-thioester substrates: propionylACP (12, the native thioester of SxtA AOS), propionylCoA (the native thiol of most characterized AOSs), and truncated substrates propionyl-pantetheine (14) and propionyl-SNAC (15). As shown in Figure 2, the simplification of this substrate readily decreased the yield of ketone product 8 in the AOS reaction. In reactions with propionyl-SNAC (15), the most truncated electrophile accepted as a substrate, less than one percent of the product was generated in compared to reactions with propionyl-CoA (10).

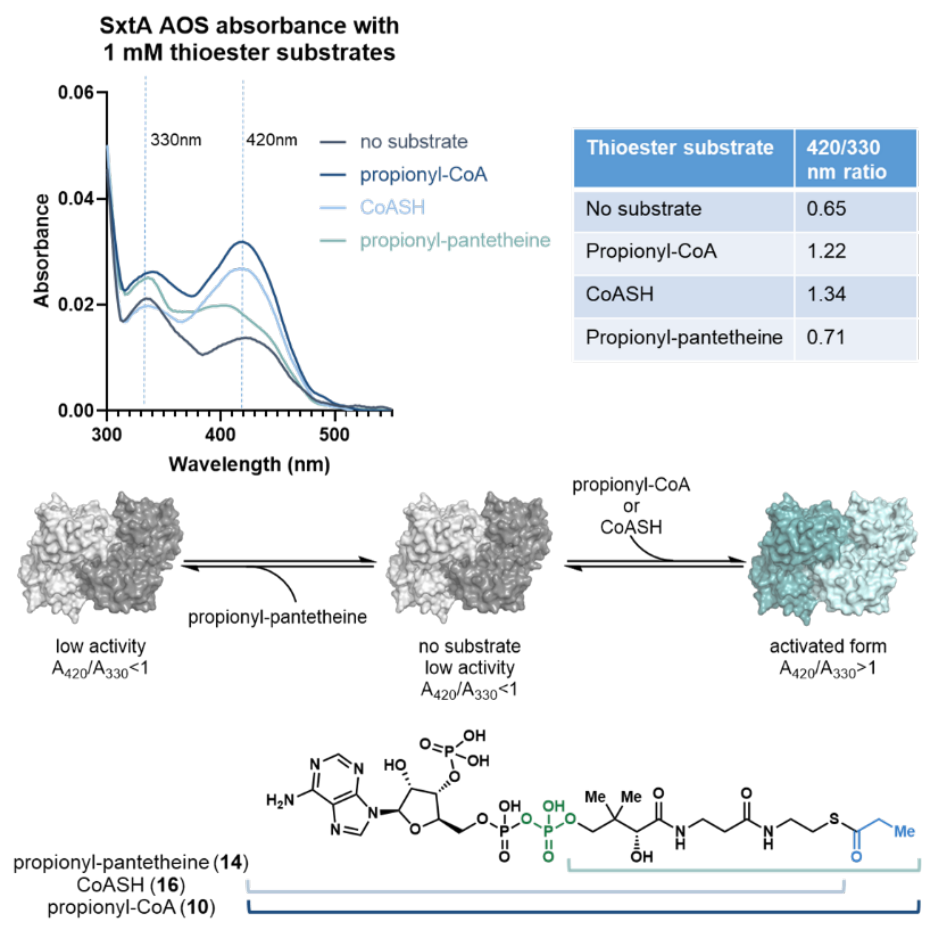

Figure 3 | PLP-absorbance changes observed in SxtA AOS with three thioester substrates in two PLP-based peaks-proposed inactive form at $330 \mathrm{~nm}$ and activated form at $420 \mathrm{~nm}$. The equilibrium is shown in the middle scheme, with observed absorbance spectra with each thioester substrate in the top left and the individual ratios of the two peaks shown in the top right. At the bottom, brackets show the structure of each acyl-substrate used in this experiment.
To further interrogate which portion of coenzyme $A$ was playing a role in modulating this reaction, we sought to investigate the impact of compounds on the PLP absorbance spectra. In these experiments, the UV-vis absorbance from 300 to 550 $\mathrm{nm}$ of $20 \mu \mathrm{M}$ enzyme was measured in the presence of $1 \mathrm{mM}$ thioester substrate (Figure 3 ). In samples containing SxtA AOS, two peaks associated with the PLP absorbance spectra are prominent, one at 330 $\mathrm{nm}$ and the other at $420 \mathrm{~nm}$. Analysis of SxtA AOS purified with no added thioester, the $330 \mathrm{~nm}$ peak was most prominent. When propionyl-CoA (10) was added and the mixture incubated for five minutes, the ratio between the $330 \mathrm{~nm}$ and $420 \mathrm{~nm}$ peaks was perturbed, with the $420 \mathrm{~nm}$ peak becoming the highest intensity peak. Because addition of this close analog of the native ACP substrate results in an increase of the $420 \mathrm{~nm}$ peak, we propose that the 420 $\mathrm{nm}$ peak is the active conformation of the enzyme that will proceed with productive catalysis. An alternative explanation is that this peak shift was due to consumption of L-Arg that had co-purified with the enzyme. To discriminate between these two scenarios, an experiment in which CoA salt was added to SxtA AOS was performed (Figure 3). This experiment reveals a similarly altered peak ratio, favoring the the proposed active form of the enzyme, despite this mixture lacking the acyl group required for product formation. This experiment provided evidence that some portion of the CoA substrate was causing a change of the electronics around PLP, rather than being a function of a chemical reaction. To further probe which portion of the thiol induced this change, $1 \mathrm{mM}$ propionyl-pantetheine was added to purified enzyme. This thioester did not induce the same change, as $330 \mathrm{~nm}$ remained the prominent peak. Comparing the results obtained from these three substrates, we hypothesize that the striking differences in the PLP-spectra in the presence of CoA-containing substrates indicate that some portion of the diphosphate group, sugar moiety, or nucleoside base is essential to accessing the catalytically active conformation of the enzyme. With the western-most phosphate group, sugar and base not included in the native propionyl-ACP substrate (12), we hypothesized that the eastern phosphodiester group was the moiety inducing the increased product formation. Furthermore, we hypothesized that specific interactions between the eastern phosphodiester group (shown in green, Figure 3 ) of CoA and SxtA AOS induce a conformational change that is critical for productive catalysis. To probe this idea, a 
homology model of the SxtA AOS domain was generated by the Phyre2 server based on the crystal structure of the related AOS 2-amino-3-ketobutyrate CoA ligase (PDB:1FC4, Figure 4). ${ }^{28}$ The position of the acyl substrate was modeled based on the structure of succinyl-CoA bound in 5-aminolevulinate synthase (PDB:2BWO). ${ }^{29}$ This was the only available crystal structure with an acyl-CoA substrate docked, and no structures have been published showing interactions between an ACP and AOS. Examination of the region proximal to phosphodiester group in question revealed two positive residues, an Arg and a Lys, anticipated to engage in stabilizing interactions with the substrate. To test this hypothesis, sitedirected mutagenesis was performed to access variants in which either residue was substituted with alanine. These variants along with wild type enzyme were evaluated in reactions with L-Arg and either propionyl-CoA or propionyl-pantetheine. These two substrates were used as propionyl-CoA contains the eastern phosphodiester group, while propionylpantetheine does not. As such, we anticipated that if the Lys and Arg residues were interacting with this phosphodiester group, then the increase in activity typically observed with propionyl-CoA would not be as significant. Reactions containing $20 \mu \mathrm{M}$ SxtA AOS or variant, $2 \mathrm{mM}$ L-Arg, and $0.5 \mathrm{mM}$ propionyl-CoA or propionyl-pantetheine in buffer were incubated overnight and product formation was measured by LC-MS. The extent to which the activity is attenuated between propionyl-CoA and propionyl-pantetheine in the $\mathrm{R} 142 \mathrm{~A}$ variant is less than in the wild type enzyme, suggesting that this residue is indeed involved in the phosphodiester interaction (Figure 4, chart).

With support for the importance of the eastern phosphodiester in gating catalysis, we envisioned a synthetic mimic of Nature's substrates could enable a productive reaction without the need for stoichiometric CoA or carrier protein-bound substrate. First, a series of auxiliary substrates were synthesized, including those found in Table 1. Next, the efficacy of $0.5 \mathrm{mM}$ of each auxiliary was evaluated in reactions of $2 \mathrm{mM}$ L-arginine and $0.5 \mathrm{mM}$ propionyl-SNAC. The amount of product formed in reactions with synthetic mimic added was compared to the amount of product formed in reactions with propionyl-SNAC alone. Both the mimic without the phosphate and with an unprotected phosphate group showed no increase in reactivity. This was unsurprising as the mimic without the phosphate was not able to form the hydrogen bonds proposed to increase reactivity, and the

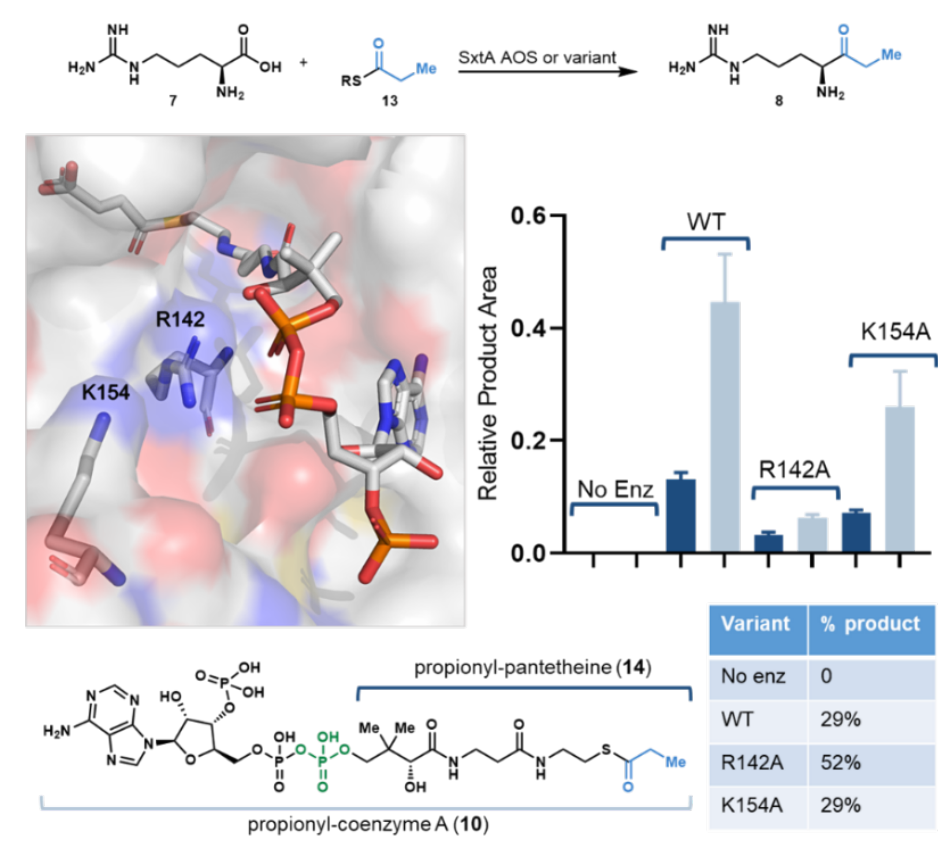

Figure 4 | Native reactivity of propionyl-pantetheine and propionyl-CoA with Ala variants of residues though to be involved in CoA binding. At top, the reaction scheme. At left, a SxtA AOS model showing the propionylCoA and the two residues substituted: Lys154 and Arg142. At right, a graph demonstrating the change in activity when each residue is varied to Ala. At bottom, brackets showing the substrates involved.

unprotected phosphate mimic added negative charge density not found in the native substrate. Only the mimic containing the protected phosphotriester moiety (entry 5, Table 1) showed increased product formation. Not only does this provide further evidence that the phosphate is essential for activity, as the same mimic without the phosphate does not induce the increase in activity, but it also provides support for the idea that a mimic can be used to improve activity, negating the need for expensive coenzyme $A$ substrates.

Table 1 | Reactions with wild-type SxtA AOS enzyme, demonstrating propionyl-SNAC's activity improves with a methylated phosphodiester mimic, demonstrating the possibility of improving reactivity without utilizing an expensive CoA substrate.

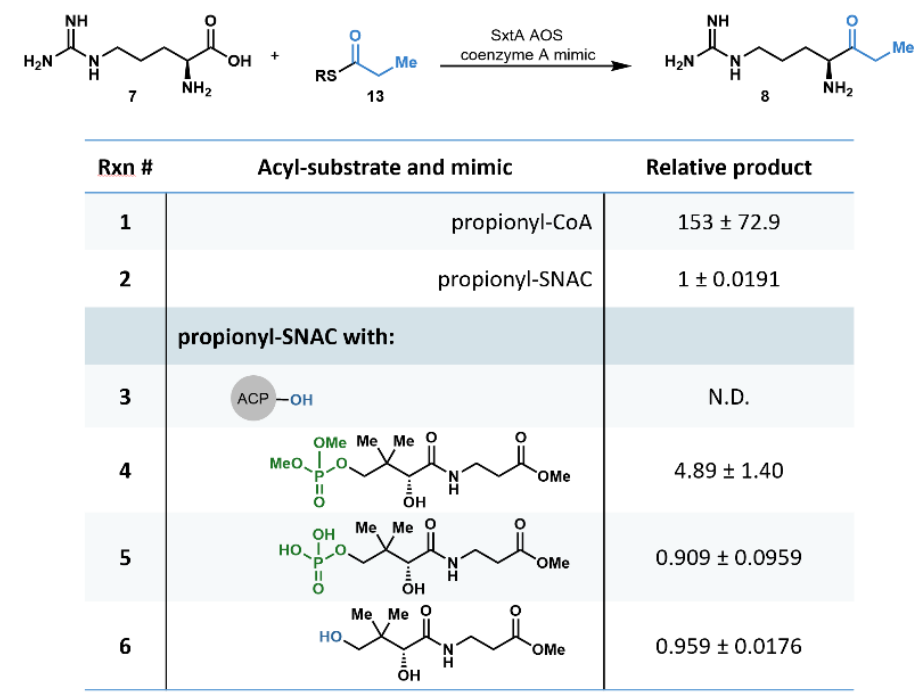




\section{Conclusions}

AOS enzymes are attractive candidates for biocatalysis, however, these enzymes naturally require acyl-CoA substrates or carrier protein-bound substrates for productive cataylsis, making these catalysts unsuitable for preparative-scale reactions. To overcome this challenge, we sought to identify the key protein-acyl substrate interactions and devise an approach to circumventing this gatekeeping mechanism. We have demonstrated that coenzyme $A$ has critical interactions with the AOS, specifically with the eastern phophodiester group, and that synthetic mimics containing this group can serve as additives that avoid the need for expensive coenzyme $A$ substrates. This work provides evidence that a mimic can be used to turn the switch of activity, and provides an avenue for the application of AOSs as useful biocatalyts for the transformation of $\alpha$-amino acids.

\section{ASSOCIATED CONTENT}

\section{Supporting Information}

The Supporting Information is available free of charge on the ACS Publications website at DOI: $\mathbf{x x}$ Supplemental figures and methods (PDF)

\section{AUTHOR INFORMATION}

\section{Corresponding Author}

Alison R. H. Narayan - Department of Chemistry, Life Sciences Institute, and Program in Chemical Biology, University of Michigan, Ann Arbor, Michigan 48109, United States; orcid.org/0000-0001-82900077; Email: arhardin@ umich.edu

\section{Authors}

Sarah E. Ackenhusen - Program in Chemical Biology and Life Sciences Institute, University of Michigan, Ann Arbor, Michigan 48109, United States orcid.org/0000-0002-7412-575X

Ye Wang - Department of Chemistry, Life Sciences Institute, and Program in Chemical Biology, University of Michigan, Ann Arbor, Michigan

Stephanie W. Chun - Department of Chemistry and Life Sciences Institute, University of Michigan, Ann Arbor, Michigan 48109, United States

\section{Notes}

The authors declare no competing financial interests.

\section{ACKNOWLEDGMENTS}

This research was supported by funds from the University of Michigan Life Sciences Institute, University of Michigan Department of Chemistry, and the National Institutes of Health R35 GM124880. S.E.A. was supported by the National Science Foundation Graduate Research Fellowship Program and the National Institutes of Health Microfluidics in Biomedical Sciences Training Program.

\section{REFERENCES}

(1) Blaskovich, M. A. T. Unusual Amino Acids in Medicinal Chemistry. J. Med. Chem. 2016, 59, 10807-10836.

(2) El-Faham, A.; Albericio, F. Peptide Coupling Reagents, More than a Letter Soup. Chem. Rev. 2011, 111, 6557-6602.

(3) Carpino, L. A.; Abdel-Maksoud, A. A.; Ionescu, D.; Mansour, E. M. E.; Zewail, M. A. 1,1-Dioxonaphtho[1,2-b]Thiophene-2Methyloxycarbonyl (a-Nsmoc) and 3,3Dioxonaphtho[2,1-b]Thiophene-2Methyloxycarbonyl ( $\beta$-Nsmoc) AminoProtecting Groups. J. Org. Chem. 2007, 72, 1729-1736.

(4) Paul, A.; Seidel, D. a-Functionalization of Cyclic Secondary Amines: Lewis Acid Promoted Addition of Organometallics to Transient Imines. J. Am. Chem. Soc. 2019, 141, 8778-8782.

(5) Isidro-Llobet, A.; Álvarez, M.; Albericio, F. Amino Acid-Protecting Groups. Chem. Rev. 2009, 109, 2455-2504.

(6) Stigers, K. D.; Koutroulis, M. R.; Chung, D. M.; Nowick, J. S. Fmoc: A More Soluble Analogue of the 9-Fluorenylmethoxycarbonyl Protecting Group. J. Org. Chem. 2000, 65, 3858-3860. Chen, M.; Liu, C.-T.; Tang, Y. Discovery and Biocatalytic Application of a PLP-Dependent Amino Acid $\mathrm{y}$-Substitution Enzyme That Catalyzes C-C Bond Formation. J. Am. Chem. Soc. 2020, 142, 10506-10515.

Rocha, J. F.; Pina, A. F.; Sousa, S. F.; Cerqueira, N. M. F. S. A. PLP-Dependent Enzymes as Important Biocatalysts for the Pharmaceutical, Chemical and Food Industries: A Structural and Mechanistic Perspective. Catal. Sci. Technol. 2019, 9 4864-4876.

Du, Y. L.; Ryan, K. S. Pyridoxal PhosphateDependent Reactions in the Biosynthesis of Natural Products. Nat. Prod. Rep. 2019, 36, 430-457.
Eliot, A. C.; Kirsch, J. F. Pyridoxal Phosphate Enzymes: Mechanistic, Structural, and Evolutionary Considerations. Annu. Rev. 
Biochem. 2004, 73, 383-415.

(11) Savile, C. K.; Janey, J. M.; Mundorff, E. C.; Moore, J. C.; Tam, S.; Jarvis, W. R.; Colbeck, J. C.; Krebber, A.; Fleitz, F. J.; Brands, J.; et al. Biocatalytic Asymmetric Synthesis of Chiral Amines from Ketones Applied to Sitagliptin Manufacture. Science. 2010, 329, 305-309.

(12) Simon, R. C.; Richter, N.; Busto, E.; Kroutil, W. Recent Developments of Cascade

Reactions Involving $\omega$-Transaminases. ACS Catal. 2014, 4, 129-143.

(13) Kelly, S. A.; Pohle, S.; Wharry, S.; Mix, S.; Allen, C. C. R.; Moody, T. S.; Gilmore, B. F. Application of $\omega$-Transaminases in the Pharmaceutical Industry. Chem. Rev. 2018, 118, 349-367.

(14) Slabu, I.; Galman, J. L.; Lloyd, R. C.; Turner, N. J. Discovery, Engineering, and Synthetic Application of Transaminase Biocatalysts. ACS Catal. 2017, 7, 8263-8284.

(15) Yoshimura, T.; Esaki, N. Amino Acid Racemases: Functions and Mechanisms. J. Biosci. Bioeng. 2003, 96, 103-109.

(16) Chun, S. W.; Narayan, A. R. H. Biocatalytic Synthesis of a-Amino Ketones. Synlett 2019, 30, 1269-1274.

(17) Ikushiro, H.; Islam, M. M.; Okamoto, A.; Hoseki, J.; Murakawa, T.; Fujii, S.; Miyahara, I.; Hayashi, H. Structural Insights into the Enzymatic Mechanism of Serine

Palmitoyltransferase from Sphingobacterium Multivorum. J. Biochem. 2009, 146, 549-562.

(18) Ikushiro, H.; Hayashi, H.; Kagamiyama, H. Reactions of Serine Palmitoyltransferase with Serine and Molecular Mechanisms of the Actions of Serine Derivatives as Inhibitors. Biochemistry 2004, 43, 1082-1092.

(19) Hunter, G. A.; Ferreira, G. C. Pre-SteadyState Reaction of 5-Aminolevulinate Synthase: Evidence for a Rate-Determining Product Release. J. Biol. Chem. 1999, 274, 12222-12228.

(20) Fanica-Gaignier, M.; Clement-Metral, J. 5Aminolevulinic-Acid Synthetase of Rhodopseudomonas Spheroides Y. Kinetic Mechanism and Inhibition by ATP. Eur. J. Biochem. 1973, 40, 19-24.

(21) Ploux, O.; Breyne, O.; Carillon, S.; Marquet, A. Slow-Binding and Competitive Inhibition of 8-Amino-7-Oxopelargonate Synthase, a Pyridoxal-5'-Phosphate-Dependent Enzyme Involved in Biotin Biosynthesis, by Substrate and Intermediate Analogs. Kinetic and Binding Studies. Eur. J. Biochem. 1999, 259, 63-70.
Webster, S. P.; Campopiano, D. J.; Alexeev, D.; Alexeeva, M.; Watt, R. M.; Sawyer, L.;
Baxter, R. L. Characterisation of S-Amino-7Osononanoate Synthase: A Bacterial PLPDependent, Acyl CoA Condensing Enzyme. Biochem. Soc. Trans. 1998, 26, S268.

Ikushiro, H.; Fujii, S.; Shiraiwa, Y.; Hayashi, H. Acceleration of the Substrate $\mathrm{Ca}$

Deprotonation by an Analogue of the Second Substrate Palmitoyl-CoA in Serine Palmitoyltransferase. J. Biol. Chem. 2008, 283, 7542-7553.

(24) Ploux, O.; Marquet, A. The 8-Amino-7Oxopelargonate Synthase from Bacillus Sphaericus. Purification and Preliminary Characterization of the Cloned Enzyme Overproduced in Escherichia Coli. Biochem. J. 1992, 283, 327-331.

(25) Kubota, T.; Shimono, J.; Kanameda, C.; Izumi, Y. The First Thermophilic a-Oxoamine Synthase Family Enzyme That Has Activities of 2-Amino-3-Ketobutyrate CoA Ligase and 7Keto-8-Aminopelargonic Acid Synthase: Cloning and Overexpression of the Gene from an Extreme Thermophile, Thermus Thermophilus, and Cha. Biosci. Biotechnol. Biochem. 2007, 71, 3033-3040.

Alexeev, D.; Alexeeva, M.; Baxter, R. L.; Campopiano, D. J.; Webster, S. P.; Sawyer, L. The Crystal Structure of 8-Amino-7Oxononanoate Synthase: A Bacterial PLPDependent, Acyl-CoA-Condensing Enzyme. J. Mol. Biol. 1998, 284, 401-419.

(27) W. Chun, S.; E. Hinze, M.; A. Skiba, M.; R. H. Narayan, A. Chemistry of a Unique Polyketide-like Synthase. J. Am. Chem. Soc. 2018, 140, 2430-2433.

(28) Schmidt, A.; Sivaraman, J.; Li, Y.; Larocque, R.; Barbosa, J. A. R. G.; Smith, C.; Matte, A.; Schrag, J. D.; Cygler, M. Three-Dimensional Structure of 2-Amino-3-Ketobutyrate CoA Ligase from Escherichia Coli Complexed with a PLP-Substrate Intermediate: Inferred Reaction Mechanism. Biochemistry 2001, 40, 5151-5160.

(29) Astner, I.; Schulze, J. O.; Van Den Heuvel, J.; Jahn, D.; Schubert, W. D.; Heinz, D. W. Crystal Structure of 5-Aminolevulinate Synthase, the First Enzyme of Heme Biosynthesis, and Its Link to XLSA in Humans. EMBO J. 2005, 24, 3166-3177. 